\title{
Analytical and Numerical Study for MHD Radiating Flow over an Infinite Vertical Surface Bounded by a Porous Medium in Presence of Chemical Reaction
}

\author{
S. Ahmed ${ }^{1}$ and K. Kalita ${ }^{2 \dagger}$ \\ ${ }^{1,2}$ Heat Transfer and Fluid Mechanics Research, Department of Mathematics and Computing, \\ Rajiv Gandhi University, Rono Hills, Itanagar 791112, Arunachal Pradesh, India
}

†Corresponding Author Email: heat_mass@yahoo.in

(Received September 6, 2012; accepted December 13, 2012)

\begin{abstract}
The study of non-linear MHD flow with heat and mass transfer characteristics of an incompressible, viscous, electrically conducting and Newtonian fluid over a vertical oscillating porous plate embedded in a porous medium in presence of homogeneous chemical reaction of first order and thermal radiation effects have been analyzed. The fluid considered here is a gray, absorbing/emitting radiation, but a non-scattering medium. At timet $>0$, the plate temperature and concentration levels near the plate raised linearly with timet. The dimensionless governing coupled, non-linear boundary layer partial differential equations are solved by an efficient, accurate, and extensively validated and unconditionally stable finite difference scheme of the Crank-Nicolson type as well as by the Laplace Transform technique. An increase in porosity parameter $(\mathrm{K})$ is found to depress the fluid velocities and shear stress in the regime. Also it has been found that, when the conduction-radiation $(\mathrm{R})$ increased, the fluid velocities as well as temperature profiles were decreased. It has been found that, when the chemical reaction parameter $\left(\mathrm{C}_{\mathrm{r}}\right)$ increased, the fluid velocities as well as concentration profiles were decreased. Applications of the study arise in materials processing and solar energy collector systems.
\end{abstract}

Keywords: Laplace technique, MHD, Absorbing/emitting radiation, Porosity, Chemical reaction.

\section{NOMENCLATURE}

$\begin{array}{ll}A & \text { a constant } \\ \bar{a} & \text { absorption coefficient } \\ C_{P} & \text { specific heat at constant pressure } \\ C_{r} & \text { chemical reaction coefficient } \\ \bar{C} & \text { species concentration } \\ \bar{C}_{\infty} & \text { species concentration in the free stream } \\ \bar{C}_{w} & \text { species concentration at the surface } \\ D & \text { chemical molecular diffusivity } \\ \text { erfc } & \text { complementary error function } \\ e r f & \text { error function } \\ G r_{m} & \text { mass Grashof number } \\ G r & \text { thermal Grashof number } \\ g & \text { acceleration due to gravity } \\ K_{r} & \text { porosity of the porous medium } \\ M & \text { hartmann number parameter } \\ N u & \text { Nusselt number } \\ P r & \text { Prandtl number } \\ q_{r} & \text { radiative heat flux } \\ R_{a} & \text { thermal radiation } \\ S c & \text { Schmidt number } \\ \bar{T} & \text { temperature }\end{array}$

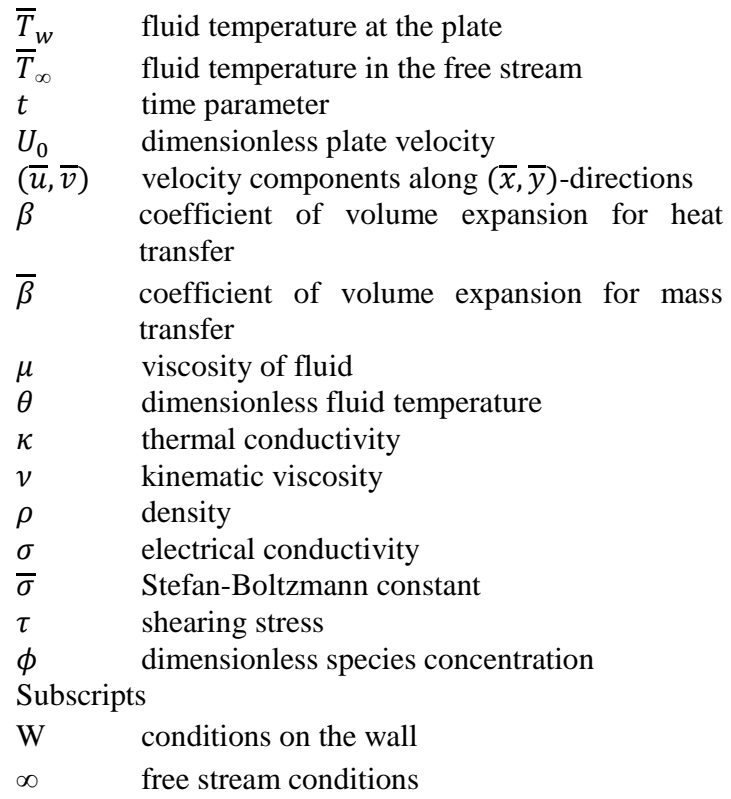




\section{INTRODUCTION}

The study of heat and mass transfer with chemical reaction is of great practical importance to engineers and scientists because of its almost universal occurrence in many branches of science and engineering. In particular, the study of chemical reaction, heat and mass transfer with heat radiation is of considerable importance in chemical and hydrometallurgical industries. A reaction is said to be first-order if the rate of reaction is directly proportional to the concentration itself. In many chemical processes, a chemical reaction occurs between a foreign mass and a fluid in which a plate is moving. These processes take place in numerous industrial applications, e.g., polymer production, manufacturing of ceramics or glassware, and food processing Cussler (1998). Chambre and Young (1958) analyzed the diffusion of chemically reactive species in a laminar boundary layer flow. Vajravelu (1986) studied the exact solution for hydrodynamic boundary layer flow and heat transfer over a continuous, moving horizontal flat surface with uniform suction and internal heat generation/absorption. Chamkha (2003) presented an analytical solution for heat and mass transfer by laminar flow of a Newtonian, viscous, electrically conducting fluid and heat generation/absorption.

On the other hand, flow through a porous medium have numerous engineering and geophysical applications, for example, in chemical engineering for filtration and purification process; in agriculture engineering to study the underground water resources; in petroleum technology to study the movement of natural gas, oil and water through the oil reservoirs. In view of these applications, many researchers have studied MHD free convective heat and mass transfer flow in a porous medium; some of them are Raptis and Kafoussias (1982), Sattar (1983) and Kim (2004). Jaiswal and Soundalgekar (2001) obtained an approximate solution to the problem of an unsteady flow past an infinite vertical plate with constant suction and embedded in a porous medium with oscillating plate temperature. The unsteady flow through a highly porous medium in the presence of radiation was studied by Raptis and Perdikis (2004). Sahin (2008) investigated the effect of transverse periodic permeability oscillating with time on the heat transfer flow of a viscous incompressible fluid through a highly porous medium bounded by an infinite vertical porous plate, by means of series solution method. Sahin (2010) studied the effect of transverse periodic permeability oscillating with time on the free convective heat transfer flow of a viscous incompressible fluid through a highly porous medium bounded by an infinite vertical porous plate subjected to a periodic suction velocity.

If the temperature of surrounding fluid is rather high, radiation effects play an important role and this situation does not exist in space technology. In such cases one has to take into account the effect of thermal radiation and mass diffusion. Boundary layer flow on moving horizontal surfaces was studied by Sakiadis (1961). The effects of transversely applied magnetic field on the flow of an electrically conducting fluid past an impulsively started isothermal vertical plate was studied by Soundalgekar et al. (1979). MHD effects on impulsively started vertical infinite plate with variable temperature in the presence of transverse magnetic field were studied by Soundalgekar et al. (1981). The dimensionless governing equations were solved using Laplace transform technique. Soundalgekar and Takhar (1993) have considered the radiation free convection flow of an optically thin gray- gas past a semi- infinite vertical plate. Radiation effects on mixed convection along isothermal vertical plate were studied by Hossain and Takhar (1996). In all above studies, the stationary vertical plate is considered. Raptis and Perdikis (1999) studied the effects of thermal radiation and free convection flow past a moving vertical plate. The governing equations were solved analytically. Also, Sahin and Liu (2010) analyzed the effects of mixed convection and mass transfer of three-dimensional oscillatory flow of a viscous incompressible fluid past an infinite vertical porous plate in presence of transverse sinusoidal suction velocity oscillating with time and a constant free stream velocity. Singh (2011) investigated the flow of fluid through porous medium bounded by vertical channel with slip-flow condition and in the presence of thermal radiation and the fluid is of optically thin with relatively low-density. Recently, Sahin (2011) investigated the effects of radiation and chemical reaction on a steady mixed convective heat and mass transfer past an infinite vertical permeable plate with constant suction taking into account the induced magnetic field. A study of unsteady laminar hydro magnetic flow and heat transfer in a porous channel with temperature-dependent properties was presented by Chamkha (2001). Sahin and Kalita (2012) investigated the effects of porosity and magneto hydrodynamic on a horizontal channel flow of a viscous incompressible electrically conducting, Newtonian and radiating fluid through a porous medium in the presence of thermal radiation and transverse magnetic field. Jaiswal and Soundalgekar (2001) obtained an approximate solution to the problem of an unsteady flow past an infinite vertical plate with constant suction and embedded in a porous medium with oscillating plate temperature. Kumar and Verma (2011) studied the problem of an unsteady flow past an infinite vertical permeable plate with constant suction and transverse magnetic field with oscillating plate temperature.

Magneto hydrodynamic mixed free-forced heat and mass convective steady incompressible laminar boundary layer flow of a gray optically thick electrically conducting viscous fluid past a semi-infinite vertical plate for high temperature and concentration differences have studied by Emad and Gamal (2005). Orhan and Kaya (2008) investigated the mixed convection heat transfer about a permeable vertical plate in the presence of magneto and thermal radiation effects using the Keller box scheme, an efficient and accurate finitedifference scheme. Ghosh et al. (2009) considered an exact solution for the hydro magnetic natural convection boundary layer flow past an infinite vertical flat plate under the influence of a transverse magnetic field with magnetic induction effects and the transformed ordinary differential equations are solved exactly. On the other hand, Ghosh and Bég (2008) for unsteady convection in porous media, Bég et al. (2009) 
for coupled species and heat diffusion in nonlinear porous media (using a network electrical simulator), Zueco and Bég (2009) for hydro magnetic gas flow from a two-dimensional wedge in porous media. Muthucumaraswamy and Janakiraman (2006) studied the thermal radiation effects on flow past an impulsively started infinite vertical plate with uniform temperature and variable mass diffusion in the presence of transverse applied magnetic field. The governing equations are solved by the Laplace-transform technique. Rajput and Kumar (2012) considering the radiation effects on MHD flow past an impulsively started vertical plate with variable heat and mass transfer by Laplace transform technique. Ahmed and Kalita (2012) presented the magneto hydrodynamic transient convective radiative heat transfer in an isotropic, homogenous porous regime adjacent to a hot vertical plate using the Laplace transform technique. Such a study has not appeared in the literature and constitutes an important addition to the area of porous media convection studies in presence of transverse magnetic field. Chamkha et al. (2002) considered the natural convection flow from an inclined, semi-infinite, impermeable flat plate embedded in a variable porosity porous medium due to solar radiation and in the presence of an externally applied magnetic field. Chamkha (1997) presented the model for Darcian and non-Darcian effects of the porous medium and the Hall effects of magneto hydrodynamics. It is assumed that the magnetic Reynolds number is small so that the induced magnetic field is neglected. The flow is assumed unsteady, laminar, and incompressible.

In this paper, we consider the effects of chemical reaction as well as magnetic field on the heat and mass transfer of Newtonian fluids over an infinite vertical oscillating plate with variable mass diffusion. The magnetic field is imposed transversely to the plate. The temperature and concentration of the plate is oscillating with time about a constant non-zero mean value. The conservation equations are normalized and then solved using both the Laplace Transform technique and stable finite difference scheme of the Crank-Nicolson type. Excellent agreement is obtained between both analytical and numerical methods.

\section{Mathematical ANALYSIS}

Thermal radiation and mass transfer effects on unsteady MHD flow of a viscous incompressible fluid past along a vertical oscillating plate with variable temperature and also with variable mass diffusion in the presence of transverse applied magnetic field and chemical reaction of first order have been studied. The $\bar{x}$ axis is taken along the plate in the vertical upward direction and the $\bar{y}$ axis is taken normal to the plate. Initially it is assumed that the plate and fluid are at the same temperature $\bar{T}_{\infty}$ in the stationary condition with concentration level $\bar{C}_{\infty}$ at all the points. At time, $\bar{t}>0$ the plate is given an oscillatory motion in its own plane with velocity $U_{0} \cos (\bar{\omega} \bar{t})$. At the same time the plate temperature is raised linearly with time $\bar{t}$ and also mass is diffused from the plate linearly with time. A transverse magnetic field of uniform strength $B_{0}$ is assumed to be applied normal to the plate. The induced magnetic field and viscous dissipation is assumed to be negligible as the magnetic Reynolds number of the flow is taken to be very small. The fluid considered here is gray, absorbing/emitting radiation but a non-scattering medium. Then by usual Boussinesq's approximation, the unsteady flow is governed by the following equations.

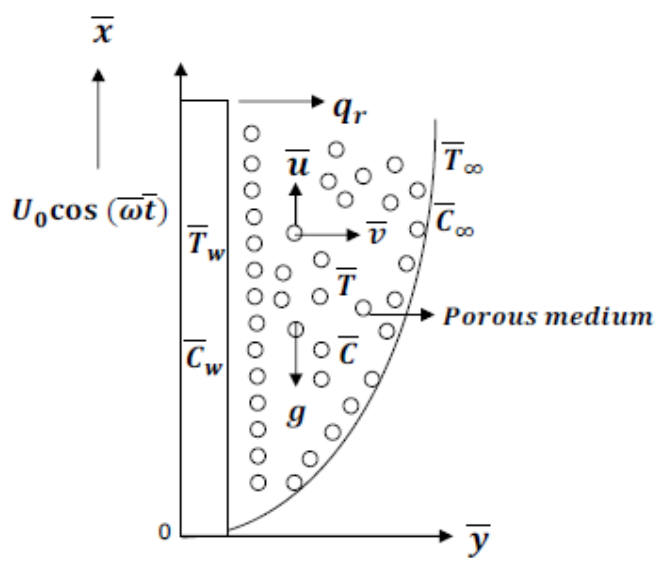

Fig. 1. Physical configuration and coordinate system

$$
\begin{gathered}
\frac{\partial \bar{u}}{\partial \bar{t}}=g \beta\left(\bar{T}-\bar{T}_{\infty}\right)+g \bar{\beta}\left(\bar{C}-\bar{C}_{\infty}\right)+v \frac{\partial^{2} \bar{u}}{\partial \bar{y}^{2}}- \\
\frac{\sigma B_{0}^{2}}{\rho} \bar{u}-\frac{v}{\bar{K}_{r}} \bar{u} \\
\rho C_{P} \frac{\partial \bar{T}}{\partial \bar{t}}=\kappa \frac{\partial^{2} \bar{T}}{\partial \bar{y}^{2}}-\frac{\partial q_{r}}{\partial \bar{y}} \\
\frac{\partial \bar{C}}{\partial \bar{t}}=D \frac{\partial^{2} \bar{C}}{\partial \bar{y}^{2}}-\bar{C}_{r}\left(\bar{C}-\bar{C}_{\infty}\right)
\end{gathered}
$$

The initial and boundary conditions are:

$$
\begin{aligned}
& \bar{t} \leq 0: \bar{u}=0, \bar{T}=\bar{T}_{\infty}, \bar{C}=\bar{C}_{\infty} \forall y \\
& \bar{t}>0: \bar{u}=U_{0} \cos (\bar{\omega} \bar{t}), \bar{T}=\bar{T}_{\infty}+\left(\bar{T}_{w}-\bar{T}_{\infty}\right) A \bar{t} \\
& \bar{C}=\bar{C}_{\infty}+\left(\bar{C}_{w}-\bar{C}_{\infty}\right) A \bar{t} \text { at } y=0 \\
& \bar{t}>0: \bar{u} \rightarrow 0, \bar{T} \rightarrow \bar{T}_{\infty}, \bar{C} \rightarrow \bar{C}_{\infty} \text { as } y \rightarrow \infty
\end{aligned}
$$

The local radiant absorption for the case of an optically thin gray gas ((2004), (2006), (2012)) is expressed as

$$
\frac{\partial q_{r}}{\partial \bar{y}}=-4 \overline{a \sigma}\left(\bar{T}_{\infty}^{4}-\bar{T}^{4}\right)
$$

Where $\bar{\sigma}$ and $\bar{a}$ are the Stefan-Boltzmann constant and the Mean absorption coefficient, respectively. Following (2004), (2006), (2012) and others, we assume that the temperature differences within the flow are sufficiently small so that $\bar{T}^{4}$ can be expressed as a linear function of $\bar{T}$ after using Taylor's series to expand $\bar{T}^{4}$ about the free stream temperature $\bar{T}_{\infty}$ and neglecting higher-order terms. This results in the following approximation: 
S. Ahmed and K. Kalita / JAFM, Vol. 6, No. 4, pp. 597-607, 2013.

$\bar{T}^{4} \cong 4 \bar{T}_{\infty}^{3} \bar{T}-3 \bar{T}_{\infty}^{4}$

$\rho C_{P} \frac{\partial \bar{T}}{\partial \bar{t}}=\kappa \frac{\partial^{2} \bar{T}}{\partial \bar{y}^{2}}-16 \overline{a \sigma} \bar{T}_{\infty}^{3}\left(\bar{T}-\bar{T}_{\infty}\right)$

Introducing the following non-dimensional quantities:

$y=\frac{u_{0} \bar{y}}{v}, \quad u=\frac{\bar{u}}{u_{0}}, \quad \theta=\frac{\bar{T}-\bar{T}_{\infty}}{\bar{T}_{w}-\bar{T}_{\infty}}, \quad \phi=\frac{\bar{C}-\bar{C}_{\infty}}{\bar{C}_{w}-\bar{C}_{\infty}}$,

$S c=\frac{v}{D}, \operatorname{Pr}=\frac{\rho v C_{P}}{\kappa}, \quad G r=\frac{v g \beta\left(\bar{T}_{w}-\bar{T}_{\infty}\right)}{u_{0}^{3}}$,

$G r_{m}=\frac{v g \bar{\beta}\left(\bar{C}_{w}-\bar{C}_{\infty}\right)}{u_{0}^{3}}, t=\frac{u_{0}^{2} \bar{t}}{v}, \quad M=\frac{\sigma B_{0}^{2} v}{\rho u_{0}^{2}}$,

$R_{a}=\frac{16 \bar{a} v \bar{\sigma} \bar{T}_{\infty}^{3}}{\kappa u_{0}^{2}}, \omega=\frac{\bar{\omega} v}{u_{0}^{2}}, \quad C_{r}=\frac{v \bar{C}_{r}}{u_{0}^{2}}$,

$A=\frac{u_{0}^{2}}{v}, \quad K_{r}=\frac{\bar{K}_{r} u_{0}^{2}}{v^{2}}$

Using the transformations Eq. (8), the non-dimensional forms of Eq. (1), Eq. (3) and Eq. (7) are

$\frac{\partial u}{\partial t}=\frac{\partial^{2} u}{\partial y^{2}}+G r \theta+G r_{m} \phi-\left(M+K_{r}^{-1}\right) u$

$\frac{\partial \theta}{\partial t}=\frac{1}{P r} \frac{\partial^{2} \theta}{\partial y^{2}}-\frac{R_{a}}{P r} \theta$

$\frac{\partial \phi}{\partial t}=\frac{1}{S c} \frac{\partial^{2} \phi}{\partial y^{2}}-C_{r} \phi$

The corresponding initial and boundary conditions are transformed to:

$$
\begin{aligned}
& t \leq 0: u=0, \theta=0, \phi=0 \quad \forall y \\
& y>0: u=\cos (\omega t), \theta=t, \phi=t \text { at } y=0 \\
& y>0: u \rightarrow 0, \theta \rightarrow 0, \phi \rightarrow 0 \text { as } y \rightarrow \infty
\end{aligned}
$$

\section{Method OF SOLUTION}

The unsteady, non-linear, coupled partial differential Eq. (9) to Eq. (11) along with their boundary conditions Eq. (12) have been solved analytically using usual Laplace transform technique and the solutions for hydro magnetic flow in the presence of radiation and first order chemical reaction are obtained as follows:

$$
\begin{aligned}
& \theta(y, t)=\left(\frac{t}{2}+\frac{y P r}{4 \sqrt{R_{a}}}\right) \mathrm{e}^{y \sqrt{R_{a}}} \operatorname{erfc}(\eta \sqrt{\operatorname{Pr}}+ \\
& \left.\sqrt{\frac{R_{a} t}{P r}}\right)+\left(\frac{t}{2}-\frac{y P r}{4 \sqrt{R_{a}}}\right) \mathrm{e}^{y \sqrt{R_{a}}} \operatorname{erfc}(\eta \sqrt{\operatorname{Pr}}- \\
& \left.\sqrt{\frac{R_{a} t}{P r}}\right)
\end{aligned}
$$

$\phi(y, t)=\left(\frac{t}{2}+\frac{\eta \sqrt{S c t}}{2 \sqrt{C_{r}}}\right) \mathrm{e}^{2 \eta \sqrt{C_{r} t S c}} \operatorname{erfc}(\eta \sqrt{S c}+$

$\left.\sqrt{C_{r} t}\right)+\left(\frac{t}{2}-\frac{\eta \sqrt{S c t}}{2 \sqrt{K_{r}}}\right) e^{-2 \eta \sqrt{K_{r} t S c}} \operatorname{erfc}(\eta \sqrt{S c}-$

$\left.\sqrt{C_{r} t}\right)$

$$
\begin{aligned}
& u(y, t)=\frac{1}{4} e^{i \omega t}\left[e^{y \sqrt{N+i \omega}} \operatorname{erfc}\{\eta+\right. \\
& \sqrt{(N-i \omega) t}\}+e^{-y \sqrt{N+i \omega}} \operatorname{erfc}\{\eta- \\
& \sqrt{(N+i \omega) t}\}]+\frac{1}{4} e^{-i \omega t}\left[e^{y \sqrt{N-i \omega}} \operatorname{erfc}\{\eta+\right. \\
& \sqrt{(N+i \omega) t}\}+e^{-y \sqrt{N-i \omega}} \operatorname{erfc}\{\eta- \\
& \sqrt{(N-i \omega) t}\}]-\frac{Q}{2}\left[e^{y \sqrt{M}} \operatorname{erfc}(\eta+\right. \\
& \left.\sqrt{N t})+e^{-y \sqrt{M}} \operatorname{erfc}(\eta-\sqrt{N t})\right]+ \\
& B\left[\left(\frac{t}{2}+\frac{y}{4 \sqrt{N}}\right) e^{y \sqrt{M}} \operatorname{erfc}(\eta+\sqrt{N t})+\left(\frac{t}{2}-\right.\right. \\
& \left.\left.\frac{y}{4 \sqrt{N}}\right) e^{-y \sqrt{M}} \operatorname{erfc}(\eta-\sqrt{N t})\right]+ \\
& \frac{E}{2} e^{C t}\left[e^{y \sqrt{N-C}} \operatorname{erfc}\{\eta+\right. \\
& \sqrt{(N-C) t}\}+e^{-y \sqrt{M-C}} \operatorname{erfc}\{\eta- \\
& \sqrt{(N-C) t}\}+\frac{G}{2} e^{e t}\left[e^{y \sqrt{N+e}} \operatorname{erfc}\{\eta+\right. \\
& \sqrt{(N+e) t}\}]+e^{-y \sqrt{N+e}} \operatorname{erfc}\{\eta- \\
& \sqrt{(N+e) t}\}]+\frac{E}{2} e^{e t}\left[e^{y \sqrt{R_{a}}} \operatorname{erfc}\{\eta \sqrt{\operatorname{Pr}}+\right. \\
& \left.\left.\sqrt{\frac{R_{a} t}{P r}}\right\}+e^{-y \sqrt{R_{a}}} \operatorname{erfc}\left\{\eta \sqrt{P r}-\sqrt{\frac{R_{a} t}{P r}}\right\}\right]+ \\
& D \theta(y, t)-\frac{E}{2} e^{-C t}\left[\mathrm{e}^{y \sqrt{R_{a}-C P r}} \operatorname{erfc}\{\eta \sqrt{\operatorname{Pr}}+\right. \\
& \left.\sqrt{\left(\frac{R_{a}}{P r}-C\right) t}\right\}+e^{-y \sqrt{R_{a}-C P r}} \operatorname{erfc}\{\eta \sqrt{\operatorname{Pr}}- \\
& \left.\left.\sqrt{\left(\frac{R_{a}}{P r}-C\right) t}\right\}\right]+F \phi(y, t)+\operatorname{Gerfc}(y \sqrt{S c})- \\
& \frac{G}{2}\left[e^{y \sqrt{e S c}} \operatorname{erfc}\{\eta \sqrt{S c}+\right. \\
& \left.\sqrt{e t}\}+e^{-y \sqrt{e s c}} \operatorname{erfc}\{\eta \sqrt{S c}-\sqrt{e t}\}\right]
\end{aligned}
$$

where $\eta=\frac{y}{2 \sqrt{t}}, \quad b=\frac{G r}{P r-1}, C=\frac{R_{a}-N}{P r-1}, d=\frac{G r_{m}}{S c-1}, e=$ $\frac{N}{S c-1}, N=M+K_{r}^{-1}, Q=\frac{G r(P r-1)}{\left(R_{a}-N\right)^{2}}+\frac{G r_{m}(S c-1)}{M^{2}}, B=$ $\frac{M\left(G r+G r_{m}\right)-R_{a} G r_{m}}{N\left(R_{a}-N\right)}, D=\frac{G r}{R_{a}-N}, E=\frac{G r(P r-1)}{\left(R_{a}-N\right)^{2}}, F=$ $\frac{G r_{m}}{N}, G=G r_{m}\left(\frac{S c-1}{N}\right)^{2}$.

\section{SKIN FRICTION}

The boundary layer produces a drag force on the plate due to the viscous stresses which are developed at the wall. The viscous stress at the surface of the plate is given by

$$
\begin{aligned}
& \tau=-\left[\frac{\partial u(y, t)}{\partial y}\right]_{y=0}=\frac{1}{2}\left[e ^ { i \omega t } \left\{\frac{e^{-(N+i \omega) t}}{\sqrt{\pi t}}+\right.\right. \\
& \sqrt{(N+i \omega) t} \operatorname{erf}(\sqrt{(N+i \omega) t})\}+ \\
& e^{-i \omega t}\left\{\frac{e^{-(N-i \omega) t}}{\sqrt{\pi t}}+\right. \\
& \sqrt{(N-i \omega) t} \operatorname{erf}(\sqrt{(N-i \omega) t})\}]+ \\
& e^{-C t}\left[\frac{e^{-(N-C) t}}{\sqrt{\pi t}}+\sqrt{N-C} \operatorname{erf}(\sqrt{(N-C) t})\right]+ \\
& G e^{e t}+E\left[\sqrt{\frac{P r}{\pi t}} e^{-R_{a} t / P r}+\sqrt{R_{a}} \operatorname{erf}\left(\sqrt{\frac{R_{a} t}{P r}}\right)\right]- \\
& D\left[t \sqrt{R_{a}} \operatorname{erf}\left(\sqrt{\frac{R_{a} t}{P r}}\right)+\right.
\end{aligned}
$$




$$
\begin{aligned}
& \left.\sqrt{\frac{t P r}{\pi}} e^{-R_{a} t / P r}+\frac{P r}{2 \sqrt{R_{a}}} \operatorname{erf}\left(\sqrt{\frac{R_{a} t}{P r}}\right)\right]- \\
& E e^{C t}\left[\sqrt{\frac{P r}{\pi t}} e^{-R_{a} t / P r}+\right. \\
& \left.\sqrt{R_{a}-C P r} \operatorname{erf}\left(\sqrt{\frac{R_{a} t}{P r}}\right)\right]+G \sqrt{\frac{S c}{\pi t}}+2 F \sqrt{\frac{t S c}{\pi}}- \\
& G e^{e t}\left[\sqrt{\frac{S c}{\pi t}} e^{-e t}+\sqrt{e S c} \operatorname{erf}(\sqrt{e t})\right]
\end{aligned}
$$

\section{NUMERICAL TEChNIQUE}

In order to solve the unsteady, non-linear coupled Eq. (9) to Eq. (11) under the conditions Eq. (12), an implicit finite difference scheme of the Crank-Nicolson type has been employed. The finite difference equations corresponding to Eq. (9) to Eq. (11) are as follows:

$$
\begin{aligned}
& \frac{\left[u_{i, j}^{n+1}-u_{i, j}^{n}\right]}{\Delta t}=\frac{1}{2(\Delta \mathrm{y})^{2}}\left[u_{i, j-1}^{n+1}-2 u_{i, j}^{n+1}+u_{i, j+1}^{n+1}+\right. \\
& \left.u_{i, j-1}^{n}-2 u_{i, j}^{n}+u_{i, j+1}^{n}\right]+\frac{G r\left[\theta_{i, j}^{n+1}+\theta_{i, j}^{n}\right]}{2}+ \\
& \frac{G r_{m}\left[\phi_{i, j}^{n+1}+\phi_{i, j}^{n}\right]}{2}-\frac{\left[u_{i, j}^{n+1}+u_{i, j}^{n}\right]}{2}\left(M+K^{-1}\right) \\
& \frac{\left[\theta_{i, j}^{n+1}-\theta_{i, j}^{n}\right]}{\Delta t}= \\
& \frac{\left[\theta_{i, j-1}^{n+1}-2 \theta_{i, j}^{n+1}+\theta_{i, j+1}^{n+1}+\theta_{i, j-1}^{n}-2 \theta_{i, j}^{n}+\theta_{i, j+1}^{n}\right]}{2 \operatorname{Pr}(\Delta y)^{2}}- \\
& \frac{R_{a}\left[\theta_{i, j}^{n+1}+\theta_{i, j}^{n}\right]}{2 \operatorname{Pr}} \\
& \frac{\left[\phi_{i, j}^{n+1}-\phi_{i, j}^{n}\right]}{\Delta t}= \\
& \frac{\left[\phi_{i, j-1}^{n+1}-2 \phi_{i, j}^{n+1}+\phi_{i, j+1}^{n+1}+\phi_{i, j-1}^{n}-2 \phi_{i, j}^{n}+\phi_{i, j+1}^{n}\right]}{2 S c(\Delta y)^{2}}- \\
& \frac{C_{r}\left[\phi_{i, j}^{n+1}+\phi_{i, j}^{n}\right]}{2 S c}
\end{aligned}
$$

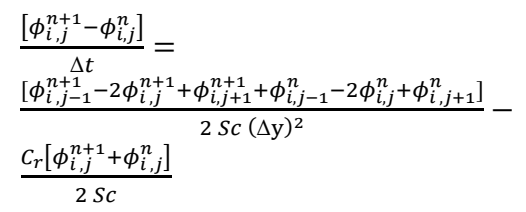

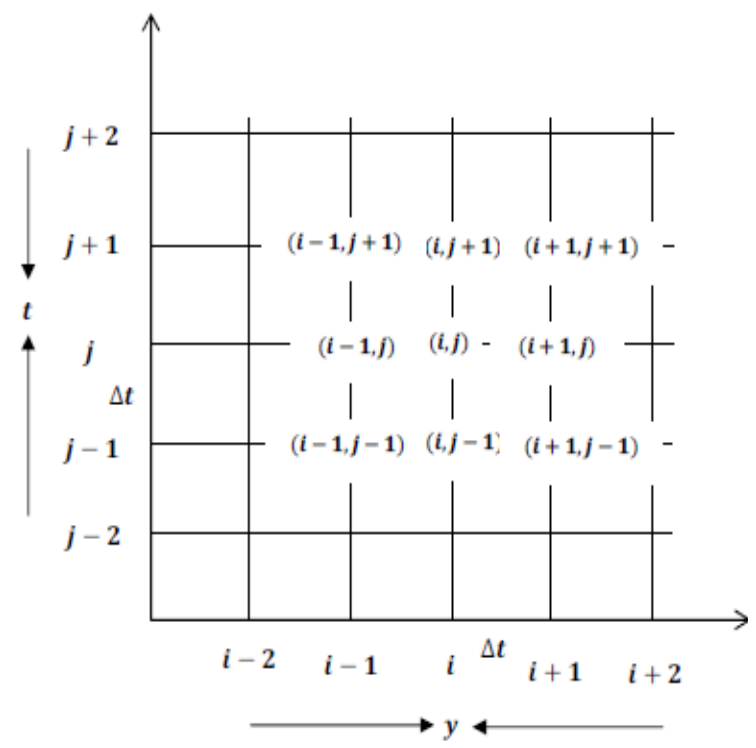

Fig. 2. Finite difference space grid

The region of integration is considered as a rectangle with sides $x_{\max }(=1)$ and $y_{\max }(=14)$, where $y_{\max }$ corresponds to $y=\infty$ which lies very well outside both the momentum and energy boundary layers. The maximum of $y$ was chosen as 14 after some preliminary investigations so that the last two of the boundary conditions (14) are satisfied within the tolerance limit $10^{-5}$. After experimenting with a few set of mesh sizes, the mesh sizes have been fixed at the level $\Delta y=0.25$ with time step $\Delta t=0.01$. In this case, the spatial mesh sizes are reduced by $50 \%$ in one direction, and later in both directions, and the results are compared. It is observed that, when the mesh size is reduced by $50 \%$ in the $y$-direction, the results differ in the fifth decimal place while the mesh sizes are reduced by $50 \%$ in $x$-direction or in both directions; the results are comparable to three decimal places.

Hence, the above mesh sizes have been considered as appropriate for calculation. The coefficients $u_{i, j}^{n}$ and $v_{i, j}^{n}$ appearing in the finite-difference equations are treated as constants in any one time step. Here $i$-designates the grid point along the $x$-direction, $j$ along the $y$-direction. The values of $u, v$ and $\theta$ are known at all grid points at $t=0$ from the initial conditions.

The computations of $u, v, \theta$ and $\phi$ at time level $(n+1)$ using the values at previous time level $(n)$ are carried out as follows: The finite difference Eq. (19) at every internal nodal point on a particular $i-$ level constitutes a tridiagonal system of equations. Such systems of equations are solved by using Thomas algorithm as discussed in Carnahan et al. (1969). Thus, the values of $\phi$ are found at every nodal point for a particular $i$ at $(n+1)^{\text {th }}$ time level. Similarly, the values of $\theta$ are calculated from Eq. (18). Using the values of $\phi$ and $\theta$ at $(n+1)^{\text {th }}$ time level in the Eq. (17), the values of $u$ at $(n+1)^{\text {th }}$ time level are found in a similar manner. Thus, the values of $\phi, \theta$ and $u$ are known on a particular $i-$ level. This process is repeated for various $i$-level. Thus the values of $\phi, \theta, u$ are known, at all grid points in the rectangular region at $(n+1)^{\text {th }}$ time level.

In a similar manner, computations are carried out by moving along the $i$-direction. After computing values corresponding to each $i$ at a time level, the values at the next time level are determined in a similar manner. Computations are repeated until the steady-state is reached. The steady state solution is assumed to have been reached, when the absolute difference between the values of $u$, as well as temperature $\theta$ and concentration $\phi$ at two consecutive time steps are less than $10^{-5}$ at all grid points.

\subsection{Stability Analysis}

The stability criterion of the finite difference scheme for constant mesh sizes are examined using Von-Neumann technique as explained by Carnahan et al. (1969). The general term of the Fourier expansion for $u, \theta$ and $\phi$ at a time arbitrarily called $t=0$, are assumed to be of the form $\exp (i \beta y)($ here $i=\sqrt{-1})$. At a later time $t$, these terms will become,

$$
\begin{aligned}
u & =H_{1}(t) \exp (i \beta y) \\
\theta & =H_{2}(t) \exp (i \beta y) \\
\phi & =H_{3}(t) \exp (i \beta y)
\end{aligned}
$$


Substituting Eq. (20) in Eq. (17) to Eq. (19) under the assumption that the coefficients $u, \theta$ and $\phi$ are constants over any one time step and denoting the values after one time step by $H_{1}{ }^{\prime}, H_{2}{ }^{\prime}$ and $H_{3}{ }^{\prime}$. After simplification, we get

$$
\begin{aligned}
& \frac{H_{1}{ }^{\prime}-H_{1}}{\Delta t}=\frac{\left(H_{2}{ }^{\prime}+H_{2}\right) G r+\left(H_{3}{ }^{\prime}+H_{3}\right) G r_{m}}{2}+\left(H_{1}{ }^{\prime}+\right. \\
& \left.H_{1}\right)\left[\frac{\cos (\beta \Delta y)-1\}}{(\Delta y)^{2}}-\frac{\left(M+K^{-1}\right)}{2}\right] \\
& \frac{H_{2}{ }^{\prime}-H_{2}}{\Delta t}=\left[\frac{\{\cos (\beta \Delta y)-1\}}{(\Delta y)^{2}}-\frac{R_{a}}{2}\right] \frac{\left(H_{2}{ }^{\prime}+H_{2}\right)}{P r} \\
& \frac{H_{3}{ }^{\prime}-H_{3}}{\Delta t}=\left[\frac{\{\cos (\beta \Delta y)-1\}}{(\Delta y)^{2}}-\frac{C_{r}}{2}\right] \frac{\left(H_{3}{ }^{\prime}+H_{3}\right)}{S c}
\end{aligned}
$$

Equations (21) to (23) can be rewritten as,

$$
\begin{aligned}
& (1+I) H_{1}{ }^{\prime}=(1-I) H_{1}+\frac{\Delta t}{2}\left[\operatorname{Gr}\left(H_{2}{ }^{\prime}+H_{2}\right)+\right. \\
& \left.G r_{m}\left(H_{3}{ }^{\prime}+H_{3}\right)\right] \\
& (1+J) H_{2}{ }^{\prime}=(1-J) H_{2} \\
& (1+L) H_{3}{ }^{\prime}=(1-L) H_{3}
\end{aligned}
$$

where

$$
\begin{aligned}
& I=[1-\cos (\beta \Delta y)] \frac{\Delta t}{(\Delta y)^{2}}+\frac{\left(M+K^{-1}\right) \Delta t}{2}, \\
& J=[1-\cos (\beta \Delta y)] \frac{\Delta t}{\operatorname{Pr}(\Delta y)^{2}}+\frac{R_{a} \Delta t}{2 P r} \\
& L=[1-\cos (\beta \Delta y)] \frac{\Delta t}{S c(\Delta y)^{2}}+\frac{C_{r} \Delta t}{2 S c}
\end{aligned}
$$

After eliminating $\mathrm{H}_{2}{ }^{\prime}$ and $\mathrm{H}_{3}{ }^{\prime}$ in Eq. (24) using Eq. (25) and Eq. (26), the resultant equation is given by,

$$
(1+I) H_{1}{ }^{\prime}=(1-I) H_{1}+H_{2} \frac{G r \Delta t}{(1+J)}+H_{3} \frac{G r_{m} \Delta t}{(1+L)}
$$

Equations (27) to (29) can be written in matrix form as follows:

$\left(\begin{array}{l}H_{1}{ }^{\prime} \\ H_{2}{ }^{\prime} \\ H_{3}{ }^{\prime}\end{array}\right)=\left(\begin{array}{ccc}\frac{1-I}{1+I} & P_{1} & P_{2} \\ 0 & \frac{1-J}{1+J} & 0 \\ 0 & 0 & \frac{1-L}{1+L}\end{array}\right)\left(\begin{array}{l}H_{1} \\ H_{2} \\ H_{3}\end{array}\right)$

where $P_{1}=\frac{G r \Delta t}{(1+I)(1+J)}$ and $P_{2}=\frac{G r_{m} \Delta t}{(1+I)(1+L)}$.

Now, for stability of the finite difference scheme, the modulus of each Eigen value of the amplification matrix does not exceed unity. Since the matrix Eq. (28) is triangular, the Eigen values are its diagonal elements. The Eigen values of the amplification matrix are $(1-I) /(1+I),(1-J) /(1+J)$ and $(1-L) /(1+$ $L)$.
Assuming that, $u$ is everywhere non-negative and $v$ is everywhere non-positive, we get

$I=2 \frac{\Delta t}{(\Delta y)^{2}} \sin ^{2}\left(\frac{\beta \Delta y}{2}\right)+\frac{\left(M+K^{-1}\right)}{2} \Delta t$

Since the real part of $A$ is greater than or equal to zero, $|(1-I) /(1+I)| \leq 1$ always. Similarly, $\mid(1-$ $J) /(1+J) \mid \leq 1$ and $|(1-L) /(1+L)| \leq 1$. Hence, the finite difference scheme is unconditionally stable. The local truncation error is $O\left(\Delta t^{2}+\Delta y^{2}\right)$ and it tends to zero as $\Delta t$ and $\Delta y$ tend to zero. Hence, the scheme is compatible. Stability and compatibility ensures convergence.

\subsection{Accuracy}

We have obtained a comprehensive range of solutions to the transformed conservation equations. To test the validity of numerical Crank Nicolson computations, we have compared the flow velocity and Concentration distributions in Tables 1 and Table 2 with the Laplace transform solutions. It is clearly seen from Table 1 and Table 2 that the results are in excellent agreement. As the accuracy of the numerical solutions is very good, the values of $u$ and $\phi$ corresponding to analytical and numerical solutions are very close to each other. Table 1 shows that the flow velocity is found to accelerate with free convection parameter $G$ from 0.0 through 5.0 to 10.0. On the other hand, increasing the Schmidt number $S c$ from 0.30 through 0.60 to 0.78 the Concentration distribution is found to depress (Table 2).

Table 1 Comparison of values of the flow velocity $(u)$ for the present results (Laplace Technique and Crank Nicolson Method) with $G r_{m}=5, R_{a}=2, K=0.2, S c=$ $0.78, M=5.0, t=0.5$ and $P r=0.71$

\begin{tabular}{|c|l|l|l|}
\hline \multicolumn{4}{|c|}{ Laplace Technique } \\
\hline \multicolumn{4}{|c|}{ Gr } \\
\hline $\mathrm{y}$ & 0.0 & \multicolumn{1}{|c|}{5.0} & 10.0 \\
\hline 0.0 & 1 & 1 & 1 \\
\hline 2.0 & 4.173150 & 4.573802 & 4.941724 \\
\hline 4.0 & 0.401362 & 0.438209 & 0.461072 \\
\hline 6.0 & 0.074019 & 0.084105 & 0.098704 \\
\hline 8.0 & 0.005317 & 0.007518 & 0.009371 \\
\hline 10.0 & 0.000852 & 0.000952 & 0.002504 \\
\hline \multicolumn{4}{|c|}{ Crank Nicolson Method } \\
\hline \multicolumn{4}{|c|}{ Gr } \\
\hline $\mathrm{y}$ & 0.0 & 1 & 5.0 \\
\hline 0.0 & 1 & 10.0 \\
\hline 2.0 & 4.174024 & 4.573983 & 4.942107 \\
\hline 4.0 & 0.402033 & 0.438571 & 0.461310 \\
\hline 6.0 & 0.075105 & 0.084791 & 0.098872 \\
\hline 8.0 & 0.005451 & 0.007619 & 0.009502 \\
\hline 10.0 & 0.000863 & 0.000971 & 0.002514 \\
\hline
\end{tabular}


Table 2 Comparison of values of the Concentration $(\phi)$ for the present results (Laplace Technique and Crank Nicolson Method) with $t=1.5, C_{r}=1$ :

\begin{tabular}{|c|c|c|c|}
\hline \multicolumn{4}{|c|}{ Laplace Technique } \\
\hline \multicolumn{4}{|c|}{ Sc } \\
\hline$y$ & 0.30 & 0.60 & 0.78 \\
\hline 0.0 & 1.000000 & 1.000000 & 1.000000 \\
\hline 2.0 & 0.347031 & 0.317402 & 0.284172 \\
\hline 4.0 & 0.118063 & 0.075043 & 0.038730 \\
\hline 6.0 & 0.024571 & 0.006527 & 0.002743 \\
\hline 8.0 & 0.003620 & 0.001209 & 0.000451 \\
\hline 10.0 & 0.000852 & 0.000372 & 0.000085 \\
\hline \multicolumn{4}{|c|}{ Crank Nicolson Method } \\
\hline \multicolumn{4}{|c|}{ Sc } \\
\hline$y$ & 0.30 & 0.60 & 0.78 \\
\hline 0.0 & 1.000000 & 1.000000 & 1.000000 \\
\hline 2.0 & 0.348051 & 0.317541 & 0.284308 \\
\hline 4.0 & 0.118131 & 0.075170 & 0.038855 \\
\hline 6.0 & 0.024618 & 0.006617 & 0.002782 \\
\hline 8.0 & 0.003704 & 0.001314 & 0.000507 \\
\hline 10.0 & 0.000867 & 0.000383 & 0.000091 \\
\hline \multicolumn{4}{|c|}{}
\end{tabular}

\section{RESUlTS AND DISCUSSION}

To gain a perspective of the physics of the flow regime, we have numerically evaluated the effects of Hartmann number $(M)$, radiation-conduction parameter $\left(R_{a}\right)$, dimensionlesstime $(t)$, porosity parameter $\left(K_{r}\right)$ and chemical reaction parameter $\left(C_{r}\right)$, on the velocity, $u$, temperature, $\theta$, shear stress function, $\left.\frac{\partial u}{\partial y}\right|_{y=0}$. Here we consider $G r=5=G r_{m}>0$ (cooling of the plate) i.e. free convection currents convey heat away from the plate in to the boundary layer, $t=0.5, R_{a}=10$ throughout the discussion.The Prandtl number $\operatorname{Pr}$ is taken for air at $20^{\circ} \mathrm{C}(\mathrm{Pr}=0.71)$, electrolytic solution $(\operatorname{Pr}=1.0)$ and water $(\mathrm{Pr}=7.0)$.

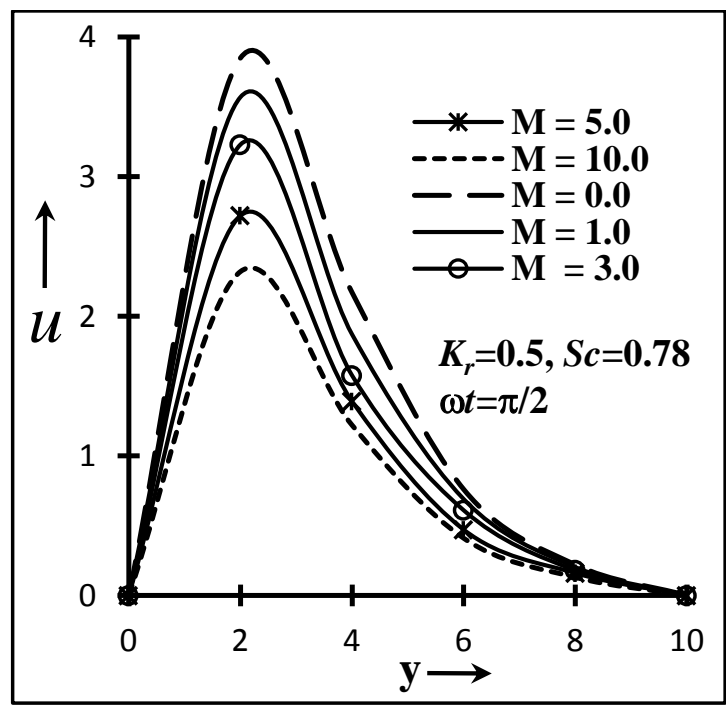

Fig. 3. Flow velocity for Hartmann number

In Fig. 3 we have presented the influence of Hartmann number square root $\mathrm{M}$ on the velocity $\mathrm{u}$ distributions with distance normal to the plate (transverse coordinate, $y$ ). The hydromagnetic term in the dimensionless equation (2.9), $-\mathrm{Mu}$ is a linear drag force term. With increasing magnetic field strength, $\mathrm{B}_{0}, \mathrm{M}$ is increased and this serves to decelerate the flow along the plate. In accordance with this, we observe in Fig. 3 that $\mathrm{u}$ profile values are strongly reduced with increasing $\mathrm{M}$. We also note that as $\mathrm{M}$ rises, the profiles decay to zero progressively for shorter distances from the plate surface. The strong inhibiting effect of magnetic field is therefore evident. Also it is noticed that the velocity distribution increases with the increase in time parameter.

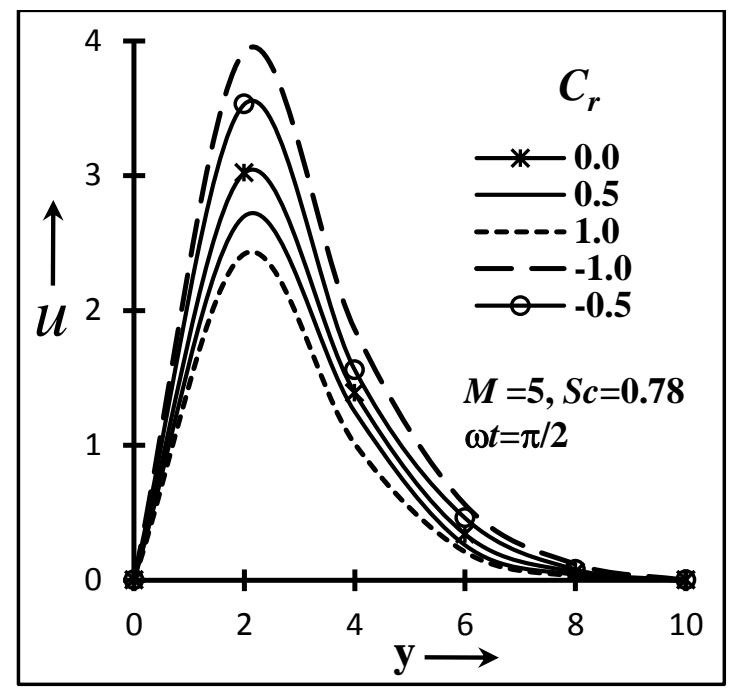

Fig. 4. Flow velocity for chemical reaction

Figures 4 and 5 display the effects of the chemical reaction parameter $C_{r}$ on the velocity $u$ and concentration $\phi$ profiles, respectively. As expected, the presence of the chemical reaction significantly affects the concentration profiles as well as the velocity profiles. It should be mentioned that the studied case is both for destructive $\left(C_{r}>0\right)$ and generative $\left(C_{r}<0\right)$ chemical reaction. In fact, as $\left(\mathrm{C}_{\mathrm{r}}>0\right)$ increases, the considerable reduction in the velocity profiles is predicted, and the presence of the peak indicates that the maximum value of the velocity occurs in the body of the fluid close to the surface but not at the surface. Also, with an increase in the chemical reaction parameter, the concentration decreases. It is evident that the increase in the chemical reaction $\mathrm{C}_{\mathrm{r}}$ significantly alters the concentration boundary layer thickness but does not alter the momentum boundary layers. Moreover, these results are observed to be opposite for the case of $\left(\mathrm{C}_{\mathrm{r}}<\right.$ $0)$.

The effect of time ( $\mathrm{t}$ ) and radiation-conduction parameter $\mathrm{R}_{\mathrm{a}}$ on spatial distribution of the dimensionless temperature function $(\theta)$ is shown in Fig. 6. Temperature is seen to decrease from a maximum value at the wall (1.0) to a minimum value with maximum distance, $y$. However with increasing time $(\mathrm{t})$ we observe that there is a clear increase in temperatures. This trend is maintained at all locations in the flow regime. At fixed time, $\mathrm{t}=0.2$, an increase in thermal radiation-conduction parameter $\left(R_{a}\right)$ is observed to strongly increase temperatures throughout the fluid with 
distance normal to the wall in the fluid regime. Larger $\left(R_{a}\right)$ values correspond to an increased dominance of thermal radiation over conduction. As such thermal radiation supplements the thermal diffusion and increases the overall thermal diffusivity of the regime since the local radiant diffusion flux model adds radiation conductivity to the conventional thermal conductivity. As a result the temperatures in the fluid regime flow are significantly increased.

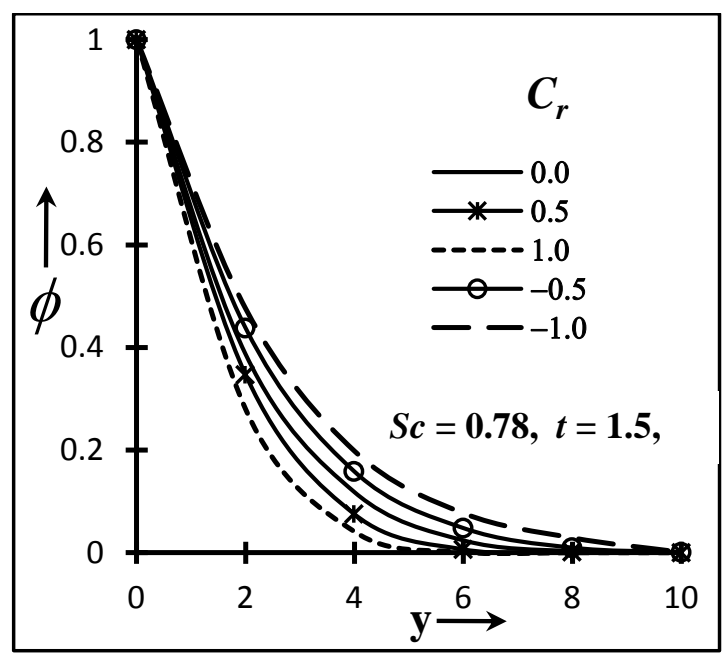

Fig. 5. Concentration for chemical reaction

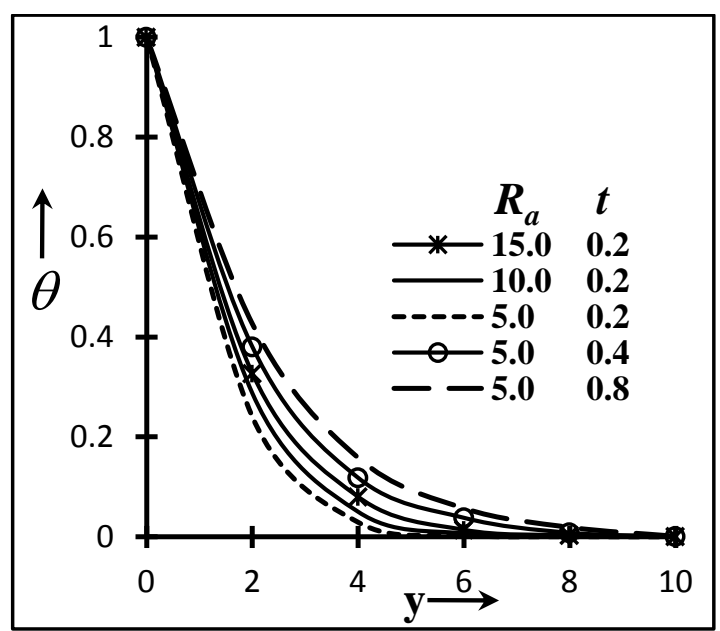

Fig. 6. Temperature for radiation and time

In Fig.7 the collective influence of the thermal radiation-conduction parameter $\left(\mathrm{R}_{\mathrm{a}}\right)$ on the shear stress function variation against Hartmann number is shown. Increasing $M$ clearly increases shear stress function $\left.\frac{\partial u}{\partial y}\right|_{y=0}$ values. Similarly in consistency with previous computations, an increase in thermal radiation also serves to acceleratethe flow which increases shear stress function values i.e. the maximum shear stress function, $\left.\frac{\partial u}{\partial y}\right|_{y=0}$, corresponds to the maximum M value (highest magnetic body force).

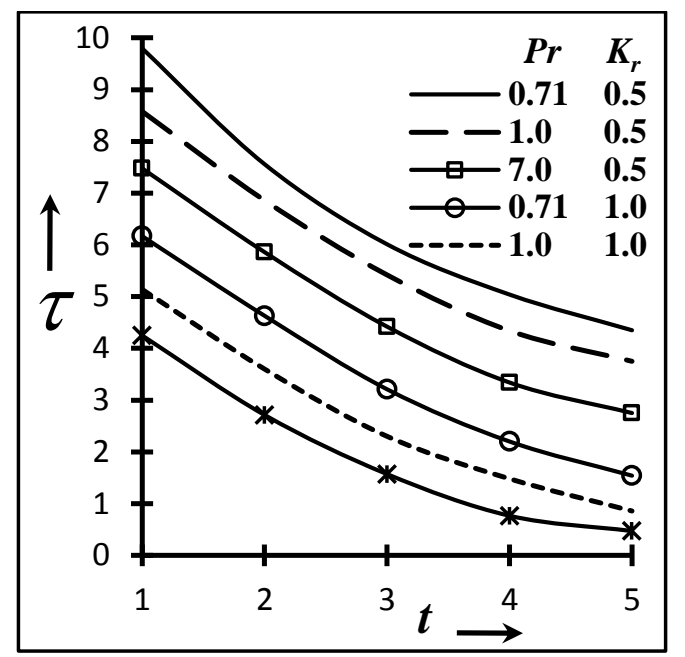

Fig. 7. Skin friction for porosity and $\mathrm{Pr}$

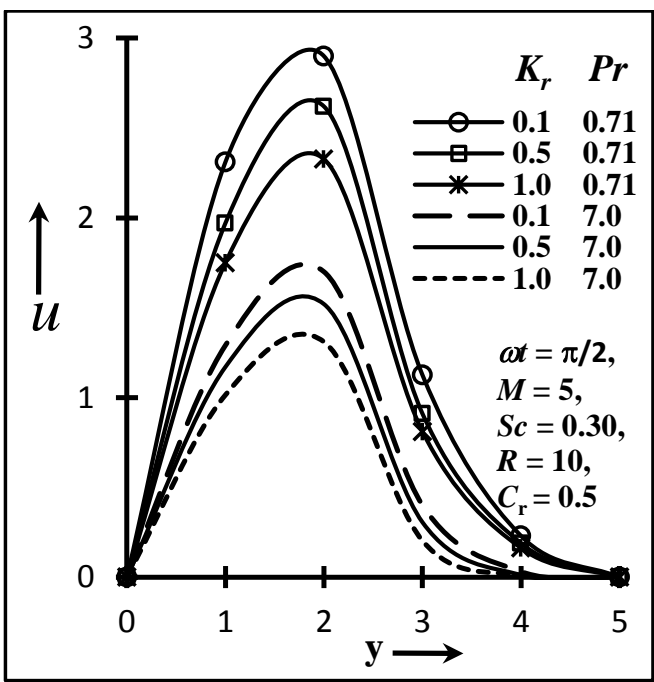

Fig. 8. Flow velocity for porosity and $\mathrm{Pr}$

Figure 8 reveals the effects of $\mathrm{K}_{\mathrm{r}}$ on the velocity profiles. The presence of a porous medium increases the resistance to flow resulting in decrease in the flow velocity. This behavior is depicted by the decrease in the velocity as $\mathrm{K}_{\mathrm{r}}$ decreases for both air and water. The magnitude of velocity for air is higher than that of water.

Figure 9 shows the skin-friction against time $\mathrm{t}$ for various values of parameters $\operatorname{Pr}$ and $\mathrm{K}_{\mathrm{r}}$. It is observed that as time passes the skin friction decreases. The skinfriction decreases with increasing permeability parameter $\mathrm{K}_{\mathrm{r}}$ for both air and water. The magnitude of the skin-friction for water is greater than that for air ( $\mathrm{Pr}$ $=0.71)$ and electrolytic solution $(\operatorname{Pr}=1.0)$.

Figure 10 illustrate the temperature $(\theta)$ profiles for different Prandtl numbersPr. The results show that the increasing Prandtlnumber results in a decrease in the thermal boundary layer and in general lower average temperature within the boundary layer. The reason is that smaller $\mathrm{Pr}$ is equivalent to the increase in the thermalconductivity of the fluid, and heat is able to diffuse away fromthe heated surface more rapidly for higher values ofPr. Therefore, in the case of smaller 
Prandtl numbers, the thermal boundary layeris thicker, and the rate of heat transfer is reduced.

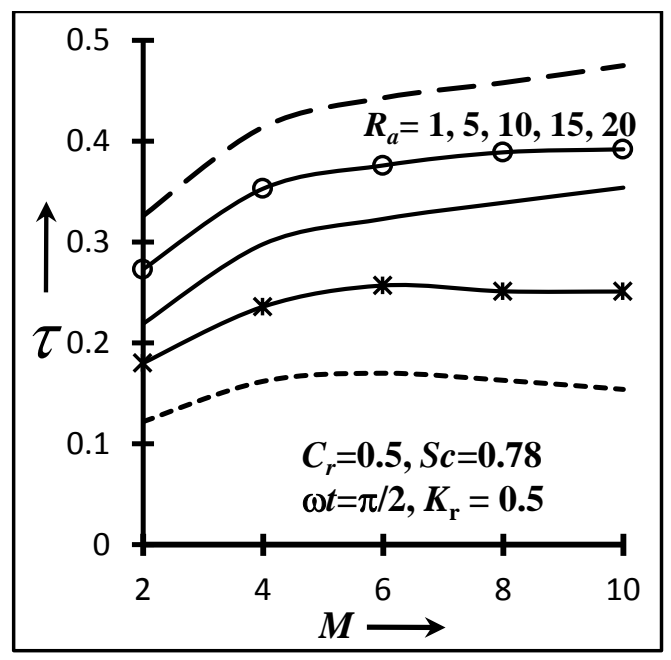

Fig. 9. Skin friction for $\mathrm{R}_{a}$ and $\mathrm{M}$ number

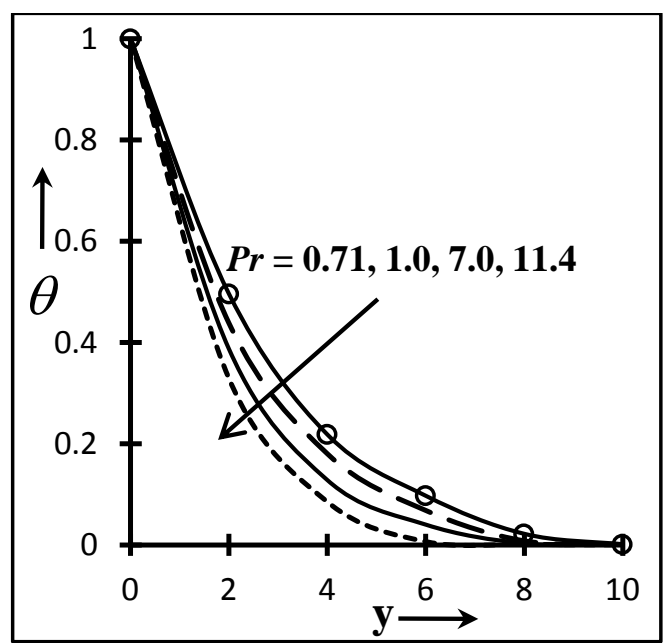

Fig. 10. Temperature distributions for $\mathrm{Pr}$

\section{CONCLUSION}

A mathematical analysis has been presented of the transient free convection-radiation magneto hydrodynamic viscous flow along an infinite vertical permeable plane under a transverse magnetic field in presence of chemical reaction. A flux model has been employed to simulate thermal radiation effects, valid for optically-thick gases. The dimensionless momentum, energy and mass conservations equations have been solved using both the Laplace transform technique and Numerical Crank Nicolson method. The study has shown that the flow is accelerated with a decrease in Hartmann number square root (M). The velocity as well as theconcentration decreases with an increase in the chemical reaction parameter. Temperature of the gas is shown to be enhanced both with the elapse of time (t) and increasing conduction-radiation $\left(R_{a}\right)$ i.e. greater thermal radiation heat transfer contribution. The currentstudy has employed a Newtonian viscous model. Presently the authors are extending this work to examine several non-Newtonian fluids of interest in glass rheological thermal processing including viscoelastic models; the results of these investigations will be communicated imminently.

\section{ACKNOWLEDGEMENTS}

Authors are highly grateful to referees for providing useful suggestions which helped us to modify this research paper. The first author thanks the University Grants Commission of India for financial support for carrying out the Research via a Research project in the year 2010-11 of No. F.5/108/2009-10/5546.

\section{REFERENCES}

Aboeldahab, E.M. and G.E. AAzzam (2005). Thermal radiation effects on magneto hydrodynamic flow past a semi-infinite vertical plate in the presence of mass diffusion. Canadian Journal of Physics, 83(3), 243-256.

Ahmed, S. (2010). Free convective transient threedimensional flow through a porous medium oscillating with time in presence of periodic suction velocity. International Journal of Applied Mathematics and Mechanics, 6, 1-16.

Ahmed, S. (2008). Transient three dimensional flow through a porous medium with transverse permeability oscillating with time. Emirate Journal for Engineering research, 1, 11-17.

Ahmed, S. and I.C. Liu (2010). Mixed convective three-dimensional heat and mass transfer flow with transversely periodic suction velocity. Int. J. Applied Mathematics and Mechanics 6, 58-73.

Ahmed, S. (2011). A study of induced magnetic field with chemically reacting and radiating fluid past a vertical permeable plate. Journal of Engineering Physics and Thermophysics,84(6), 1360-1368.

Ahmed, S. and K. Kalita (2012). A sinusoidal fluid injection/suction on MHD three-dimensional Couette flow through a porous medium in the presence of thermal radiation. Journal of Energy Heat and Mass Transfer (In Press).

Ahmed, S. and K. Kalita, (2013). Magneto hydrodynamic transient flow through a porous medium bounded by a hot vertical plate in presence of radiation: A theoretical analysis. Journal of Engineering Physics and Thermophysics, 86(1), 31-39

Aydm O. and A. Kaya (2008). Radiation effect on MHD mixed convection flow about a permeable vertical plate. Heat Mass Transfer, 45, 239-246. 
Bég, O.A., J. Zueco, T.A. Bég, H.S. Takhar and E. Kahya (2009). NSM analysis of time-dependent nonlinear buoyancy-driven double-diffusive radiative convection flow in non-Darcy geological porous media. ActaMechanica, 202,181-204.

Carnahan, B., H.A. Luther and J.O. Wilkes (1969). Applied Numerical Methods, John Wily and Sons, New York.

Chambre, P.L. and J.D. Young (1958). On the Diffusion of Chemically Reactive Species in a Laminar Boundary Layer Flow, Phys. Fluids, 1,48-54.

Chamkha, A.J. (2001). Unsteady laminar hydro magnetic flow and heat transfer in porous channel with temperature dependent properties. Int. J. Numerical Methods for Heat Fluid Flow, 11(5), 430-448.

Chamkha, A.J., C. Issa and K. Khanafer (2002). Natural convection from an inclined plate embedded in a variable porosity porous medium due to solar radiation. International journal of thermal sciences , 41(1), 73-81.

Chamkha, A.J. (1997). MHD-free convection from a vertical plate embedded in a thermally stratified porous medium with Hall effects. Applied Mathematical Modelling, 21(10), 603-609.

Chamkha, A. (2003). MHD Flow of Uniformly Stretched Vertical Permeable Surface in the Presence of Heat Generation/ Absorption and a Chemical Reaction. Int. Comm. Heat Mass Transfer, 30, 413-422.

Cussler, E.L. (1998). Diffusion, Mass Transfer in Fluid Systems, $2^{\text {nd }}$ edition, Cambridge University Press, Cambridge.

Ghosh, S.K., O.A. Bég, and J. Zueco, (2009). Hydromagnetic free convection flow with induced magnetic field effects. Physics and Astronomy, 45(2), 175-185.

Ghosh, S.K. and O.A. Bég (2008). Theoretical analysis of radiative effects on transient free convection heat transfer past a hot vertical surface in porous media. Nonlinear Analysis: Modelling and Control, 13(4), 419-432.

Hossain, M. A. and H.S. Takhar (1996). Radiation effect on mixed convection along a vertical plate with uniform surface temperature. Heat and mass transfer, 31, 243-248.

Jaiswal, B.S. and V.M. Soundalgekar (2001). Oscillating plate temperature effects on a flow past an infinite vertical porous late with constant suction and embedded in a porous medium. Heat and Mass Transfer, 37, 125-131.

Kim, Y.J. (2004). Heat and mass transfer in MHD micropolar flow over a vertical moving porous plate in a porous medium. Transport in Porous Media, 56(1), 17-37.

Kumar, A.G.V. and S.V.K. Verma (2011). Thermal radiation and mass transfer effects on MHD flow past a vertical oscillating plate with variable temperature effects variable mass diffusion. Int. Journal of Engineering, 3, 493-499.

Muthucumaraswamy, R. and B. Janakiraman (2006). MHD and radiation effects on moving isothermal vertical plate with variable mass diffusion. Theoret. Appl. Mech., 33 (1), 17-29.

Rajput, U. S. and S. Kumar (2012). Radiation effects on MHD flow past an impulsively started vertical plate with variable heat and mass transfer, Int. J. of Appl. Math. and Mech., 8(1), 66-85.

Raptis, A, and C. Perdikis (2004). Unsteady flow through a highly porous medium in the presence of radiation. Transport in Porous Media, 57, 171179.

Raptis, A. and C. Perdikis (1999). Radiation and free convection flow past a moving plate. Int. journal of Applied Mechanics and Engineering, 4, 817821.

Raptis, A. and N.G. Kafoussias (1982). Magneto hydrodynamic free convection flow and mass transfer through porous medium bounded by an infinite vertical porous plate with constant heat flux. Can. J. Phys., 60(12), 1725-1729.

Sakiadis, B.C. (1961). Boundary layer behavior on continuous solid surfaces: II boundary layer on a continuous solid flat surfaces. AiChE journal, 7 , 221-225.

Sattar, M.A. (1993). Unsteady hydromagnetic free convection flow with Hall current mass transfer and variable suction through a porous medium near an infinite vertical porous plate with constant heat flux. Int. J. Energy Research, 17, 15 . 
S. Ahmed and K. Kalita / JAFM, Vol. 6, No. 4, pp. 597-607, 2013.

Singh, K. D. (2011). Hydromagnetic forced convective oscillatory slip flow through porous medium in a vertical channel with thermal radiation.Proc. Indian Natn. Sci. Acad, 77(1), 1930.

Soundalgekar, V.M. and H.S. Takhar (1993). Radiation effects on free convection flow past a semi-infinite vertical plate. Modeling, measurement and control, 51, 31-40.

Soundalgekar, V.M., M.R. Patil, and M.D. Jahagirdar (1981). MHD stokes problem for a vertical plate with variable temperature. Nuclear Engg Des, 64, 39-42.
Soundalgekar, V. M., S. K. Gupta and N.S. Birajdar (1979). Effects of mass transfer and free convection currents on MHD Stokes problem for a vertical plate. Nuclear Engg Des, 53, 339-346.

Vajravelu, K. (1986). Hydrodynamic Flow and Heat Transfer over Continuous, Moving Porous, Flat Surface. ActaMechanica, 64,179-185.

Zueco, J. and O.A. Bég (2009). Network simulation solutions for laminar radiating dissipative magneto-gas dynamic heat transfer over a wedge in non-Darcian porous regime. Mathematical and Computer Modelling, 50,439-452. 